\title{
Assistência farmacêutica e acompanhamento farmacoterapêutico em populações chaves acometidas por tuberculose: uma revisão integrativa de literatura
}

Pharmaceutical care and Pharmacotherapeutic monitoring in braces affected by tuberculosis: an integrative literature review

Atención farmacéutica y seguimiento farmacoterapéutico en poblaciones clave afectadas por la tuberculosis: una revisión integradora de la literatura

\section{Resumo}

Esta revisão teve como objetivo apresentar a importância do assistência farmacêutica e acompanhamento farmacoterapêutico pelo profissional farmacêutico com relação as populações chaves acometidos com tuberculose. Trata-se de uma Revisão Integrativa da Literatura (RIL), sobre a assistência farmacêutica relacionada ao serviço desenvolvido pelo profissional farmacêutico. As fontes de estudos utilizadas, foram compiladas a partir das seguintes bases de dados: Biblioteca Virtual de Saúde, Literatura Latino-Americana e do Caribe em Ciências da Saúde, Scientific Electronic Library Online. A amostra desta RIL ficou então composta por um total de 13 artigos originais que preenchem os critérios previamente determinados pelo estudo, que descrevem de forma objetiva e atualizada sobre o tema. No que diz respeito às bases de dados, (9) pertenciam ao SciELO, (1) BVS, (3) LILACS. Mais um dado interessante, pois mostra a prevalência do SciELO. A assistência farmacêutica foi considerado como imprescindível para a promoção da qualidade de vida dos usuários de antirretrovirais, por meio de um acompanhamento com orientações, esclarecimentos de dúvidas, escuta ativa, apoio, aconselhamento e monitorização do tratamento farmacológico para o uso racional dos medicamentos. Espera-se que os resultados encontrados possam nortear ações para melhorias na qualidade dos serviços prestados pelos farmacêuticos diante da TB e gerar reflexões sobre o papel desse profissional no acompanhamento do paciente com tal enfermidade.

Palavras-chave: Tuberculose; Atenção farmacêutica; Coinfecção; Populações; Vulnerabilidade; HIV.

\begin{abstract}
This review aimed to present the importance of pharmaceutical care and pharmacotherapeutic monitoring by the pharmacist about key populations affected with tuberculosis. This is an Integrative Literature Review (RIL) on pharmaceutical care related to the service developed by the pharmacist. The sources of studies used were compiled from the following databases: Virtual Health Library, Latin American and Caribbean Literature on Health Sciences, Scientific Electronic Library Online. The sample of this RIL was then composed of a total of 13 original articles that meet the criteria previously determined by the study, describing the subject in an objective and updated manner. Concerning databases, (9) belonged to SciELO, (1) BVS, (3) LILACS. Another interesting fact, as it shows the prevalence of SciELO. Pharmaceutical care was considered essential to promote the quality of life of antiretroviral users, through follow-up with guidance, clarification of doubts, active listening, support, counseling, and monitoring of pharmacological treatment for the rational use of drugs. It is expected that the results found can guide actions to improve
\end{abstract}


the quality of services provided by pharmacists in the face of TB and generate reflections on the role of this professional in monitoring patients with this disease.

Keywords: Tuberculosis; Pharmaceutical attention; Coinfection; Populations; Vulnerability; HIV.

\section{Resumen}

Esta revisión tuvo como objetivo presentar la importancia de la atención farmacéutica y el seguimiento farmacoterapéutico por parte del farmacéutico en relación con las poblaciones clave afectadas por la tuberculosis. Se trata de una Revista Integrativa de Literatura (RIL) sobre la atención farmacéutica relacionada con el servicio desarrollado por el farmacéutico. Las fuentes de los estudios utilizados fueron compiladas a partir de las siguientes bases de datos: Biblioteca Virtual en Salud, Literatura Latinoamericana y del Caribe en Ciencias de la Salud, Biblioteca Científica Electrónica en Línea. La muestra de este EIR quedó luego compuesta por un total de 13 artículos originales que cumplen con los criterios previamente determinados por el estudio, describiendo el tema de manera objetiva y actualizada. En cuanto a las bases de datos, (9) pertenecían a SciELO, (1) BVS, (3) LILACS. Otro dato interesante, ya que muestra la prevalencia de SciELO. La atención farmacéutica se consideró fundamental para promover la calidad de vida de los usuarios de antirretrovirales, a través del seguimiento con orientación, aclaración de dudas, escucha activa, apoyo, asesoramiento y seguimiento del tratamiento farmacológico para el uso racional de los medicamentos. Se espera que los resultados encontrados puedan orientar acciones para mejorar la calidad de los servicios que brindan los farmacéuticos ante la TB y generar reflexiones sobre el papel de este profesional en el seguimiento de los pacientes con esta enfermedad.

Palabras clave: Tuberculosis; Atención farmacéutica; Coinfección; Vulnerabilidad; VIH.

\section{Introdução}

O Brasil ocupa o $16^{\circ}$ lugar entre os 22 países responsáveis por $80 \%$ dos casos estimados de tuberculose no mundo e é prioritário para a implementação e expansão da estratégia o tratamento para o controle da doença (BARREIRA, 2018). A Organização Mundial de Saúde (OMS) estabeleceu metas, visando à eliminação da Tuberculose para o período de 2016 a 2035, por meio da Estratégia \& Tuberculose que incluem a redução de 95\% dos óbitos e de $90 \%$ na incidência da doença, em comparação ao ano de 2015 (Bertolozzi, et al., 2020).

A Tuberculose (TB) é uma doença infectocontagiosa causada pelo Bacilo de Koch (Mycobacterium Tuberculosis) e transmissível em sua forma pulmonar. Tratável e curável, ainda se destaca mundialmente como um problema de grande relevância em saúde pública (Ferri et al.2014). Esse agravo se apresenta nas formas, pulmonar e a extrapulmonar. Em sua forma pulmonar, o contágio se dá pelo ar por meio de aerossóis, gotículas de saliva expelidas do pulmão, ou da laringe de indivíduos infectados com tuberculose ativa de via respiratória. Na forma extrapulmonar, o risco de contaminação é menor e os seus sintomas se manifestam de acordo com o órgão ou sistema acometido (Giergowicz \& Mahmud, 2020).

Segundo Giergowicz \& Mahmud (2020) no Brasil em 2014, o Sistema de Informação de Agravos de Notificação (SINAN) registrou 88.450 casos, dos quais 73.835 na forma pulmonar, 11.844 na forma extrapulmonar, e 2.720 pulmonar e extrapulmonar simultaneamente. O índice de mortalidade de 2013 foi por volta de 2,3 óbitos por 100 mil habitantes, inferior aos 2,9 óbitos por 100 mil habitantes apontados em 2003 (Jacobs \& Pinto Junior, 2020). Ainda neste contexto, segundo a Fundação Oswaldo Cruz, em 2013 no Brasil, a tuberculose permanece como quarta causa de morte por doenças infectocontagiosas e a primeira causa quando relacionado à infecção pelo Vírus da Imunodeficiência Humana (HIV) (Oliveira, et al., 2021).

Exclusão social e miséria estão diretamente relacionadas com a tuberculose, afetando principalmente pessoas submetidas a condições inadequadas de moradia, alimentação, educação e saneamento básico (Bertolozzi, et al., 2020). O abuso de álcool, drogas ilícitas e tabaco também contribuem para um maior risco de infecção e doença, assim como a coinfecção com o HIV. Desta forma, algumas populações são consideradas mais vulneráveis para a TB e vêm sendo priorizadas nas políticas e programas de controle da doença - pessoas em situação de rua, povos indígenas, pessoas que vivem com HIV, usuários de drogas, pacientes mantidos em instituições fechadas (asilos, casas de repouso, hospitais psiquiátricos e albergues), indígenas e pessoas privadas de liberdade (PPL) (Silva et al., 2019; Belardinelli et al., 2020). 
Atualmente, as políticas de saúde consideram esse controle como responsabilidade dos municípios brasileiros e reconhecem as ações do Programa de Controle da Tuberculose (PCT) como competência da Atenção Básica à Saúde (ABS) para melhoria do acesso às ações de diagnóstico e tratamento da doença (Ferri, et al., 2014).

O profissional farmacêutico é ferramenta muito importante no combate a esta doença, controlando, dispensando a medicação e orientando os pacientes, desta forma prestando assistência à equipe de saúde e Assistência Farmacêutica (AF) aos pacientes (Rodrigues, Aquino \& Medina, 2018). Esses profissionais auxiliam quanto à administração correta dos medicamentos, forma de armazenamento, possíveis interações medicamentosas, interações com alimentos, e reconhecimento de reações adversas a fim de manter a adesão ao tratamento, evitando o abandono que é fato corriqueiro neste processo, consequentemente ao passo que o paciente adere ao tratamento passa a evitar novos contágios e combater a resistência bacteriana (Da Silva \& De Lima, 2021).

O seguimento farmacoterapêutico é a prática profissional em que o farmacêutico se responsabiliza pelas necessidades do paciente relacionadas com os medicamentos, mediante a detecção, prevenção e solução de PRM de forma contínua, sistematizada e documentada, em colaboração com o próprio paciente e com os demais profissionais do sistema de saúde, com a finalidade de alcançar resultados concretos que melhorem a qualidade de vida do paciente (Da Silva, et al., 2020).

A partir do enfoque apresentado, o objetivo deste estudo é apresentar revisão integrativa sobre a importância do profissional farmacêutico com relação as populações chaves acometidos com tuberculose. Analisando a forma o perfil das populações chaves acometidas por tuberculose que fazem acompanhamento farmacoterapêutico e a importância do serviço farmacêutico nas práticas do cuidado desses pacientes.

Este artigo está estruturado em cinco seções, incluindo esta introdução. Na seção 2 apresenta-se a revisão bibliográfica. Na seção 3 são descritos os procedimentos metodológicos. Na seção 4 são apresentados e discutidos os resultados da pesquisa e, na última seção são apresentadas as principais conclusões da pesquisa.

\section{Revisão de literatura}

\subsection{Panorama epidemiológico e operacional da tuberculose (TB) no Brasil}

O gênero Mycobacterium é o único da família Mycobacteriaceae, que compreende mais de 100 espécies e são assim chamadas pois possuem ácidos micólicos em sua parede celular, o que lhes confere baixa permeabilidade, reduz a efetividade da maioria dos antibióticos e facilita sua sobrevida nos macrófagos (Muniz, et al., 2020). As espécies do gênero é o Mycobacterium tuberculosis, bacilo responsável por cerca de 98,5\% dos casos de infecção de tuberculose (De Andrade, et al., 2018).

A tuberculose (TB) é uma doença antiga - estudos de esqueletos humanos mostram que afetou humanos por milhares de anos. Sua causa permaneceu desconhecida até 24 de março de 1882, quando o Dr. Robert Koch anunciou a descoberta do bacilo responsável, posteriormente denominado Mycobacterium tuberculosis (Batista, 2021).

A Doença De acordo com o World Health Statistics divulgado pela Organização Mundial da Saúde (OMS), estima-se que no ano de 2016, no Brasil, havia 45 casos de tuberculose a cada 100.000 pessoas. A TB é uma doença transmissível que é a principal causa de problemas de saúde, uma das 10 principais causas de morte em todo o mundo e a principal causa de morte por um único agente infeccioso (WHO, 2020b).

Em 2019, cerca de 10 milhões de pessoas desenvolveram tuberculose e 1,4 milhões morreram. A tuberculose é causada pelo bacilo M. tuberculosis, que se espalha quando as pessoas que estão com tuberculose expele bactérias no ar; por exemplo, tossindo. A doença geralmente afeta os pulmões (pulmonar TB), mas também pode afetar outros locais (TB extrapulmonar) (WHO, 2020a).

TB é um doença que se mantém como importante problema de saúde pública, em especial nos países em desenvolvimento, que respondem por $95 \%$ dos casos notificados e $98 \%$ dos óbitos ocorridos no mundo. A endemia está difundida 
na população em geral, porém, existe predomínio no grupo de adultos-jovens, o que termina por afetar a economia desses países, constituindo problema médico-social permanente (Gonçalves, Cristo \& Junior, 2019).

A TB é transmitida por via aérea em praticamente todos os casos. A infecção ocorre a partir da inalação de núcleos secos de partículas contendo bacilos expelidos pela tosse, fala ou espirro do doente com tuberculose ativa de vias respiratórias (pulmonar ou laríngea) (Ramos, et al., 2018). Os doentes bacilíferos, isto é, aqueles cuja baciloscopia de escarro é positiva, são a principal fonte de infecção. Doentes de tuberculose pulmonar com baciloscopia negativa, mesmo que tenham resultado positivo à cultura, são muito menos eficientes como fontes de transmissão, embora isso possa ocorrer. As formas exclusivamente extrapulmonares não transmite a doença (Teixeira, et al., 2020).

Para o perfeito diagnóstico, o paciente deve ser encaminhado para o exame bacteriológico e radiológico. Também são analisados possíveis contatos do indivíduo com portadores de tuberculose, história de tratamento anterior para a doença e o enquadramento a um dos grupos de risco para o desenvolvimento da doença. Sendo assim, para o eficaz controle da tuberculose na sociedade é necessário realizar um diagnóstico rápido e precoce, devendo ter alta sensibilidade, alta especificidade e alto valor preditivo positivo (Winter \& Grazinoli Garrido, 2017).

\subsection{Populações chaves acometidas por tuberculose}

Um dos fatores que mais contribuem para o aumento dos índices de tuberculose, é a incidência concomitante ao HIV, além das condições de pobreza: populações marginalizadas, aglomerados carcerários (presídios), as péssimas condições de habitação, à semelhança de favelas e comunidades de extrema carência de serviços públicos básicos (saneamento básico, por exemplo) como em algumas comunidades indígenas. Condições essas que dificultam o acesso aos serviços de saúde (Gonçalves, Cristo \& Junior, 2019).

Além dos fatores relacionados ao sistema imunológico de cada pessoa e à exposição ao bacilo, o adoecimento por tuberculose, muitas vezes, está ligado às condições precárias de vida. Assim, alguns grupos populacionais podem apresentar situações de maior vulnerabilidade (Alcalde, et al.2018). A Tabela 1, a seguir indica algumas dessas populações e os seus respectivos riscos de adoecimento em comparação com a população geral

Tabela 1 - Populações chaves acometidas por tuberculose e os seus respectivos riscos de adoecimento em comparação com a população geral.

\begin{tabular}{ccc}
\hline População & Risco de adoecimento por TB* & Carga entre os casos novos* \\
\hline Indígenas & 3 & $1,0 \%$ \\
Privados de liberdade & 35 & $11,2 \%$ \\
Pessoas vivendo com o HIV & 21 & $8,5 \%$ \\
Pessoas em situação de rua & $56^{* *}$ & $2,8 \%$ \\
\hline
\end{tabular}

* Brasil (2019+); **Tbweb, SP, 2015 e Pessoa em Situação de Rua: Censo São Paulo, capital (2015).

Fonte: Adaptado de Sinan/MS e IBGE.

\subsubsection{Pessoas vivendo com HIV acometidas com Tuberculose}

Mundialmente, a última estimativa para o número de pessoas vivendo com Vírus da Imunodeficiência Humana (HIV) no mundo foi de aproximadamente 37 milhões, e que em 2017 tenham ocorridos pelo menos 1,8 milhões de novas infecções e 940 mil de óbitos entre adultos e crianças em decorrência do HIV. Em decorrência da imunossupressão causada pelo HIV, o indivíduo tem maior susceptibilidade a desenvolver doenças, caracterizadas como doenças oportunistas (Miranda et al.2020). 
Dentre essas doenças, destaca-se a tuberculose (TB), uma das mais frequentes e debilitantes infecções oportunistas. A literatura aponta evidências sobre a associação TB-HIV e a elevação da probabilidade de morte das pessoas acometidas e, consequentemente, um aumento relativo das taxas de mortalidade (Ralaizara, et al., 2020).

Devido ao risco aumentado de adoecimento por tuberculose, em toda visita da pessoa que vive com HIV aos serviços de saúde, deve ser questionada a presença de tosse e de febre, sudorese noturna ou emagrecimento, os quais, associados ou não à tosse, também podem indicar tuberculose (Alcalde, et al., 2018).

O diagnóstico precoce de infecção pelo HIV em pessoas com tuberculose e o início oportuno do tratamento antirretroviral reduzem a mortalidade. Portanto, o teste para diagnóstico do HIV (rápido ou sorológico) deve ser ofertado a toda pessoa com diagnóstico de tuberculose. Em pessoas vivendo com HIV-TB, a gravidade clínica da doença e a necessidade mais frequente de regimes sem rifampicina por interação com o tratamento antirretroviral (TARV) tornam o tratamento mais complexo. Como a rifampicina é o fármaco de maior potência tuberculostática, esses regimes podem apresentar desfechos desfavoráveis (Ralaizara, et al., 2020).

\subsubsection{Tuberculose e população indígena}

A população indígena no Brasil é composta por pessoas autodeclaradas indígenas, segundo o quesito raça/cor, definido pelo Instituto Brasileiro de Geografia e Estatística (IBGE). A doença tornou-se significativamente expressiva para os indígenas após a colonização, quando esses povos foram inseridos em um ambiente com novos agentes patogênicos, o que evoluiu a representar atualmente no Brasil uma incidência quatro vezes maior do que comparado ao nível nacional, e dez vezes superior em relação a populações não indígenas (Zorzi, et al., 2019).

Apesar disso, em 2017 foram notificados 69.569 casos novos de TB no Brasil, que corresponde a um coeficiente de incidência igual a 33,5 casos/100 mil habitantes, já na região Norte o coeficiente foi maior que a média nacional, com 42,7 casos novos/100 mil habitantes. Em relação aos povos indígenas, a incidência de TB é ainda maior em comparação a população não indígena (Paiva, et al., 2019)

Observa-se o desamparo na saúde enfrentado pelos povos indígenas, tornando-os mais suscetíveis às diversas doenças, principalmente à tuberculose. Tal contexto é resultado de vários elementos como: a situação socioeconômica, alto índice de desnutrição e baixa escolaridade (De Faria, 2020).

As condições sanitárias são evidenciadas pela falta de saneamento básico, precárias condições habitacionais e o padrão de transmissão nas aldeias. Também, há de se ressaltar os fatores culturais que interferem no atendimento, como a carência de intérpretes culturais que facilitam a troca de informações entre o paciente e o profissional responsável e a valorização da medicina tradicional desses povos. Além disso, o difícil acesso à saúde e a baixa cobertura da atenção primária são determinantes que colaboram para a perpetuação da doença na sociedade indígena (Tavares, et al., 2021).

\subsubsection{Tuberculose e população em situação de rua}

A população em situação de rua (PSR) constitui-se de um grupo heterogêneo, em situação de pobreza extrema, vínculos familiares fragilizados ou rompidos e moradia não convencional de caráter permanente ou temporário. O Brasil é um dos 22 países com alta endemicidade para tuberculose (De Queiroga, De Sá \& Gazzinelli, 2018). A Organização Mundial da Saúde estima que cerca de um quarto da população mundial seja portadora do Mycobacterium tuberculosis e 5 a $10 \%$ dos infectados desenvolvam a tuberculose ao longo da vida (Tarcisio Oliveira et al., 2021).

A ausência de moradia e as precárias condições de vida são fatores determinantes para a ocorrência da doença na PSR. Segundo estudo realizado na capital Salvador, BA, a tuberculose aparece como a terceira maior causa de adoecimento nesse grupo específico. No estado de São Paulo, estudo com base em dados de 2009 a 2013 estimou a magnitude da tuberculose, cujos 
percentuais de abandono do tratamento entre a PSR são particularmente altos, chegando a 57,3\%. Pessoas em situação de rua apresentaram uma incidência 10 a 85 vezes superior de infecções latentes por tuberculose e doença ativa, quando comparadas à população geral. Por esses motivos, o Programa Nacional de Controle da Tuberculose inclui a PSR como população prioritária (Santos, et al., 2021).

\subsubsection{Pessoas privada de liberdade acometidos com Tuberculose}

As pessoas privadas de liberdade (PPL) vivem em condições desumanas, com alto número de apenados. Entre os principais fatores que contribuem para o aumento da incidência da tuberculose em populações carcerárias, destacam-se: gênero masculino; um baixo nível de educação; vindo de uma comunidade desfavorecida; uso de drogas ilícitas; alta prevalência de infecção pelo HIV; acesso limitado a cuidados de saúde; células superlotadas, mal iluminadas e mal ventiladas; e falta de informação sobre tuberculose, tais condições aumentam 28 vezes as chances de esta população no sistema prisional, adoecer por tuberculose, quando comparada com a população geral (Da Silva, et al., 2019).

Diante desse cenário, se viu a necessidade de fortalecer o plano; no ano de 2014, foi instituída a Política Nacional de Atenção Integral à Saúde das Pessoas Privadas de Liberdade no Sistema Prisional (PNAISP); tal política dispõe sobre as condições para a adesão e a pactuação para a atenção básica de saúde no sistema prisional. A limitação na estrutura das prisões e sua dinâmica diferenciada, características que devem ser analisadas para se garantir uma assistência que garanta o cuidado integral dos detentos; algumas ações podem ser desenvolvidas nesse sentido para garantir uma resposta positiva física e biológica (Felipe, et al.,2021).

Por ser uma doença considerada de difícil controle, principalmente em locais fechados que favorecem sua transmissibilidade, como é o caso das prisões, é necessário que os profissionais de saúde que ali atuam estabeleçam um fluxo de exames e busca ativa rotineira, sempre realizando o que é preconizado nos programas nacionais de controle da TB. Ressalta-se ainda que as ações de controle da TB nas prisões não devem acontecer de forma pontual; os esforços devem ser amplos, integrando o cuidado dentro e fora das prisões, justamente por se saber que o detento continua ter contato direto com a população em geral por meio de visitas (De Brito, Pezarezi \& Dos Sasaki, 2017).

\subsection{Assistência farmacêutica a pacientes com tuberculose}

A finalidade da Assistência farmacêutica (AF) é a responsabilidade que possui na qualidade de vida da população, integrando ações de promoção, prevenção, recuperação e reabilitação da saúde. É a interação direta do farmacêutico com o usuário, visando uma farmacoterapia racional e a obtenção de resultados definidos e mensuráveis, voltados para a melhoria da qualidade de vida. Esta interação também deve envolver as concepções dos seus sujeitos, respeitadas as especificidades biopsicossociais, sob a ótica da integralidade das ações de saúde (Da Silva, et al., 2017).

A AF facilita o alcance dos resultados esperados da farmacoterapia, minimizando e/ou prevenindo o aparecimento de Problemas Relacionados com Medicamentos (PRM) e Resultados Negativos Associados a Medicação (RNM), o que reflete em um impacto positivo no sistema de saúde, pois: reduz custos por consulta de urgência e hospitalização, diminui consultas médicas e facilita e melhora a relação da unidade de saúde com o paciente, contribuindo para a adesão e persistência ao tratamento resultando, por fim, na cura da doença (Da Silva, et al., 2017).

A AF divide-se, em assistência farmacêutica ambulatorial (a qual dispensa de medicamentos diretamente aos pacientes para uso em seu domicilio) e a hospitalar (que dispensa medicamentos para a pacientes nos serviços de saúde) (Rodrigues, Aquino \& Medina, 2018). Em ambos o farmacêutico é indispensável, desde que esteja consciente de suas atribuições, determinado e capaz. Partindo dos preceitos preconizados pela AF o farmacêutico deve entender a dispensação dos medicamentos como uma grande oportunidade de interação com o paciente, uma vez que o farmacêutico será teoricamente o 
último profissional ao qual ele terá contato no decorrer de todo o processo terapêutico, desde o diagnóstico até a dispensação do medicamento, obviamente outros profissionais de saúde também poderão e devem dar o auxílio necessário a este paciente. O que não significa, portanto que este vínculo com o farmacêutico deverá ser desfeito, pelo contrário. É exatamente neste momento que ações do cuidado farmacêutico poderão ser iniciadas afinco (Nicoletti, et al., 2020).

\section{Metodologia}

Trata-se de uma Revisão Integrativa da Literatura (RIL), sobre a assistência farmacêutica relacionada ao serviço desenvolvido pelo profissional farmacêutico. Este método escolhido de revisão foi escolhido porque permite a inserção das evidências na prática clínica a partir da fundamentação do conhecimento científico, com resultados de qualidade por meio da prática baseada na evidência. Desta forma, o produto é o estado do conhecimento do tema investigado: a implementação de intervenções efetivas na prestação de cuidados e a identificação de fragilidades que poderão conduzir ao desenvolvimento de futuras investigações (Araújo, et al., 2020).

Os conteúdos abordados proporcionarão um espectro amplo sobre o tema abordado buscando um entendimento no âmbito profissional, assim como os aspectos específicos importantes direcionados ao ciclo da assistência farmacêutica na saúde de paciente que vivem com o vírus do HIV, privados de liberdade, em situação de rua e indígenas em situação de vulnerabilidade com a TB. Para nortear esta revisão, formulou-se a seguinte questão norteadora: "Quais as dificuldades e condutas/ações na assistência farmacêutica aos pacientes chaves acometidos com tuberculose?”. O desenvolvimento desse estudo será realizado em seis etapas que integram uma revisão integrativa: 1) Definição da questão de investigação; 2) Levantamento bibliográfico; 3) Definição das informações selecionadas e categorização dos estudos; 4) Avaliação do material selecionado; 5) Interpretação dos resultados; 6) Apresentação da revisão e síntese do conhecimento.

O levantamento dos referenciais bibliográficos ocorreu entre os meses de janeiro a maio de 2021. As fontes de estudos utilizadas, foram compiladas a partir das seguintes bases de dados: Biblioteca Virtual de Saúde (BVS), Literatura LatinoAmericana e do Caribe em Ciências da Saúde (LILACS), Scientific Electronic Library Online (SciELO).

Optou-se por utilizar somente artigos científicos, os quais os descritores usados para a busca, foram escolhidos após consulta ao Descritores em Ciências da Saúde (DeCS). Desta forma, utilizou-se empregou-se o operador booleano AND em conjunto com os seguintes descritores: assistência farmacêutica AND tuberculose, tuberculose AND HIV, tuberculose AND pessoas no sistema prisional, tuberculose AND situação de vulnerabilidade, tuberculose AND indígenas, tuberculose AND panorama epidemiológico.

A seleção dos artigos científicos foi realizada obedecendo rigorosamente aos critérios de inclusão e de exclusão. Inicialmente, os periódicos foram avaliados pelo título e, em seguida, pelo resumo, informações relevantes para os critérios de elegibilidade para inclusão foram os artigos completos e indexados nas bases de dados mencionadas; publicados no período entre 2012-2021; publicações somente em português; as descrições dos estudos publicados em periódicos indexados.

Os critérios de exclusões dos artigos que não compactuam com a temática; publicações repetidas, artigos com mais de 15 anos de publicação e não disponíveis na íntegra; teses e/ou dissertações, foram excluídos. A sistematização da seleção das publicações nas bases da literatura científica está apresentada em um fluxograma (Figura 1), com a descrição das etapas de busca com o quantitativo de publicações em cada uma das bases.

\section{Resultados e Discussão}

A busca bibliográfica resultou em 108 registros, incluindo 13 referências correspondentes à pesquisa avaliativa, descritivas, qualitativas, transversais, revisões bibliográficas, revisões sistemáticas, revisão integrativa e estudo clínico. Os estudos foram publicados dentro do período do ano de 2012 a 2021, no idioma português. 
A amostra desta RIL ficou então composta por um total de 13 artigos originais que preenchem os critérios previamente determinados pelo estudo, que descrevem de forma objetiva e atualizada sobre o tema, conforme apresentado na Figura 1. No que diz respeito às bases de dados, (9) pertenciam ao SciELO, (1) BVS, (3) LILACS. Mais um dado interessante, pois mostra a prevalência do SciELO.

Figura 1 - Fluxograma do processo de seleção das publicações selecionadas para compor essa RIL.

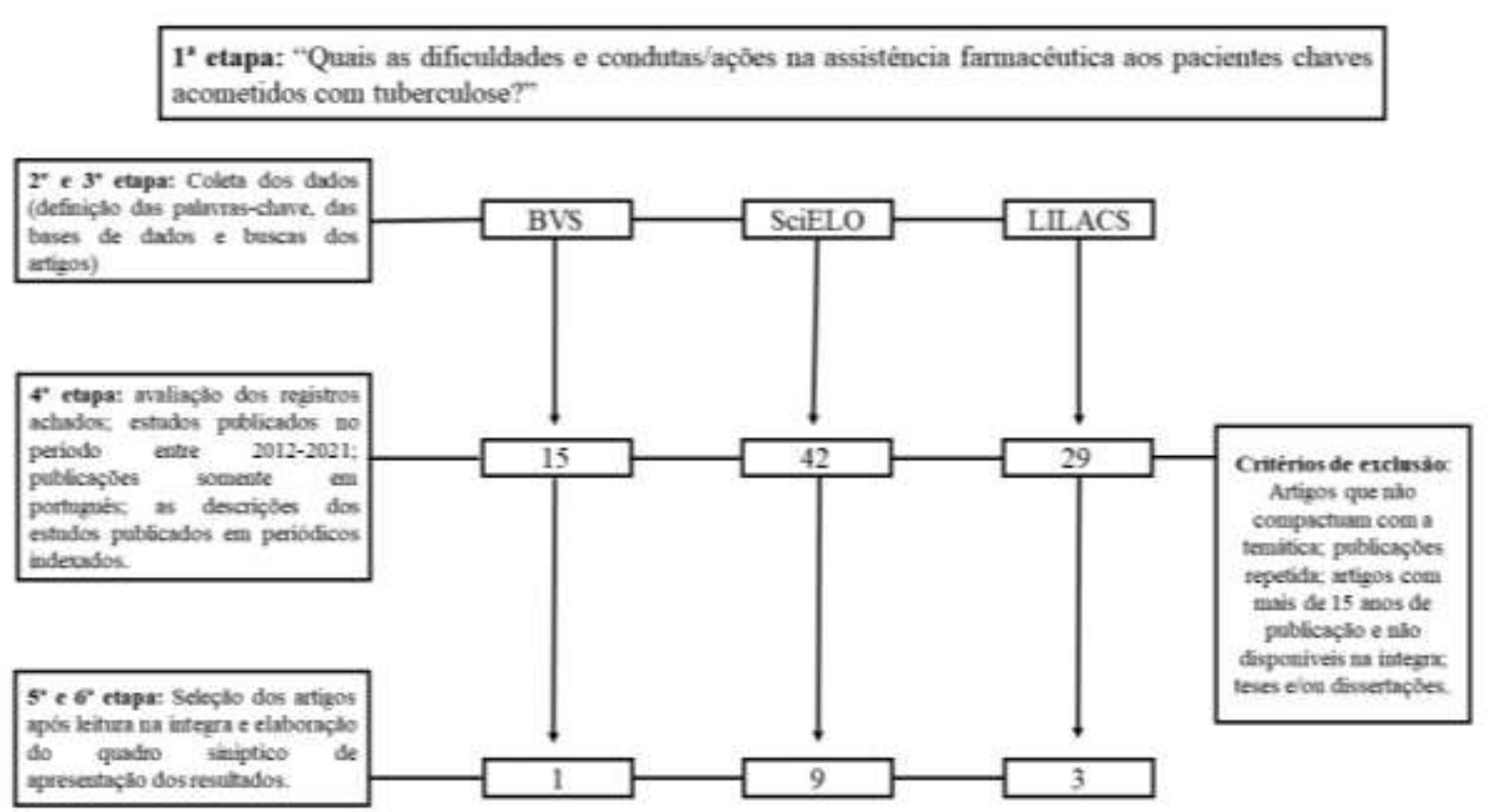

Fonte: Autores.

A presença do farmacêutico no âmbito da AF onde esta ação é responsável pela interação entre o farmacêutico e paciente, que se caracteriza como um conjunto de ações do profissional dentro do cenário da assistência farmacêutica englobando as atitudes mais coerentes para promoção à saúde de forma integrada com toda a equipe multidisciplinar. A prática é de grande importante para garantir o contato direto do farmacêutico com o usuário do medicamento uma vez que o objetivo principal se baseia na promoção de uma farmacoterapia racional, fazendo com que o paciente tenha uma melhor qualidade de vida (Nicoletti, et al., 2020).

A análise temática permitiu identificar inicialmente as dificuldades enfrentadas pelos farmacêuticos, assim como, os estratégias para melhoria na AF para a compreensão e prevenção da TB em população privada de liberdade, em situação de rua, vivem com o vírus do HIV e indígenas.

\subsection{A assistência de farmacêutica no controle da tuberculose em pessoas vivendo com HIV}

O trabalho com pessoas vivendo com HIV é contínuo, o farmacêutico é essencial nesse processo, realizando intervenções educativas, fornecendo ao paciente orientações a fim de evitar problemas relacionados aos medicamentos, algumas delas: evitar a automedicação, manter uma alimentação saudável, o prejuízo do uso de álcool e drogas ilícitas, também orientar sobre a importância de fazer uso da camisinha, sugerir adequação de doses e horários de tomadas de medicamentos agindo em conjunto com toda a equipe multidisciplinar (Rodrigues, Aquino \& Medina, 2018).

A interação TB e HIV/AIDS é capaz de acelerar a progressão de ambas as doenças, devido à imunossupressão causada pelo HIV. A coinfecção é responsável pelo aumento dos índices de mortalidade, tornando-se um desafio para a saúde pública. O 
Tabela 2 apresenta cinco artigos com ano de publicação variando de 2017-2021 e apresenta as dificuldades e condutas/ações na assistência farmacêutica de casos de TB e HIV/AIDS, com o acompanhamento farmacoterapêutico do profissional farmacêutico, a fim de prevenir a não adesão ou desistência do tratamento.

Tabela 2 - Descrição dos artigos selecionados sobre as dificuldades e condutas/ações na assistência farmacêutica aos pacientes vivendo com HIV-tuberculose.

\begin{tabular}{l} 
Referência da publicação \\
\hline \\
Silva, A. D. et al. (2020) \\
Avaliação da qualidade de vida \\
de pacientes coinfectados com \\
HIV/tuberculose em um \\
hospital do nordeste do Brasil. \\
Rev Bras Farm Hosp Serv \\
Saude, 11(2): 0346.
\end{tabular}

Alcalde, G. F. G. et al. (2018). Perfil epidemiológico de tuberculose em pacientes portadores de HIV. Rev Pre Infec e Saúde, 4:7519.

CARVALHO, W. S., \& RESENDE, N. H. (2020). Drug utilization research in coinfected patients with tuberculosis and HIV/AIDS. Revista Brasileira de Farmácia Hospitalar e Serviços de Saúde, 11(1): 0420-0420.

Resende, N. H. D. et al. (2019). Problemas relacionados ao uso de medicamentos em pacientes com tuberculose e HIV/AIDS em hospital referência. Einstein (São Paulo), 17(4):1-9.

Da Silva, I. P. et al. (2020). Problemas relacionados à farmacoterapia e intervenções farmacêuticas em indivíduos com tuberculose. Revista Brasileira de Pesquisa em Saúde/Brazilian Journal of Health Research, 22(1): 60-70.

\section{Tipo de estudo}

Dificuldades

Condutas/Ações

- O domínio físico estão diretamente relacionadas com piores condições clínicas e socioeconômicas;

- Outras comorbidades relatadas, a hipercolesterolemia e a hipertensão, depressão, insatisfação com o baixo peso corporal, medo de infectar o parceiro(a);

- O hábito de fumar, o alcoolismo e uso de drogas ilícitas.

- Provimento insuficiente de Estudo temporal, profissionais, matérias e recursos; quantitativo e - Sobrecarga de trabalho;

descritivo - Realização de atividades que não compete ao profissional.

- Os participantes do estudo apresentaram níveis de contagem de LT-CD4+ abaixo de 200 células $/ \mathrm{mm}^{3}$ e $\mathrm{CV}$ elevada, indicando imunodeficiência avançada;

Estudo transversal

- O número médio de medicamentos por paciente se manteve elevado devido aos esquemas de tratamento utilizados para tratar a coinfecção;

- Às inúmeras doenças associadas e a necessidade de profilaxia de IO.

- Alta frequência de pacientes com PRM;

- Os indivíduos coinfectados com tuberculose e HIV/AIDS maiores de 40 anos possuiram maior chance de apresentarem problemas relacionados ao uso de medicamentos durante o tratamento.

- As comorbidades mais prevalentes foram dependência química, HIV/AIDS e desnutrição.

- Interações medicamentosas e as reações adversas a medicamentos.
- Melhorias nas logísticas dos serviços de saúde; gestão em saúde; capacitação dos profissionais;

- Atuação do profissional farmacêutico no acompanhamento farmacoterapêutico e na orientação dos pacientes quanto aos esquemas utilizados.

- Atuação de agente comunitário de saúde na construção de elo entre os profissionais e a comunidade;

- Realização de mutirões de prevenção e atendimento noturno.

- Diagnóstico precoce de ambas as doenças;

- Implantação da TARV no momento oportuno;

- Capacitação dos profissionais para devida orientação aos pacientes.

- Medidas para garantir os medicamentos indicados, efetivos e seguros para sua condição clínica e a consciência da necessidade de aderir ao tratamento proposto;

- Ação importante para melhorar os resultados nos cuidados desses pacientes, evitando falha terapêutica e aparecimento de multirresistência.

- A otimização da terapia farmacológica foi parte essencial no cuidado à pessoa com TB/ HIV;

- Avaliação das prescrições medicamentosas e revisão da farmacoterapia por um profissional farmacêutico contribuem com a equipe multiprofissional de saúde na identificação de problemas farmacoterapêuticos.

Fonte: Dados da pesquisa

Silva et al. (2020), através de um estudo transversal, demonstram que os entrevistados 26 pacientes, dentre os quais $76,9 \%$ eram do gênero masculino, em sua maioria heterossexuais, solteiros, em idade produtiva (29-38 anos), sem vínculo empregatício e com condições socioeconômicas desfavoráveis. Dentre os entrevistados, 46,1\% apresentavam avançado estado 
de comprometimento imunológico. 73,1\% considerou sua saúde "boa" ou "muito boa" e $69,2 \%$ não se consideravam doentes. A qualidade de vida geral, score de zero (pior qualidade de vida) a cem (melhor qualidade de vida) teve como média 69,6 $\pm 9,1$. Quanto aos domínios, a pior média foi obtida no nível de independência $(11,1 \pm 2,6)$ e as maiores pontuações foram dos domínios espiritualidade $(15,5 \pm 3,8)$ e psicológico $(15,3 \pm 2,2)$.

Os dados obtidos no estudo de uma redução do total de casos de coinfecção HIV/TB na cidade de Americana (SP), no período total avaliado, de 2000 a 2015. Os casos de tuberculose foram mais elevados na população masculina. Também se observou que, nos dois últimos anos, houve queda no número de casos de tuberculose notificados e aumento de casos de cura da doença. A análise dos dados permitiu concluir que ocorreu uma redução de número de casos de coinfecção no período avaliado (Alcalde, et al., 2018).

Os indivíduos coinfectados com TB e HIV/AIDS maiores de 40 anos possuem maior chance de apresentarem problemas relacionados ao uso de medicamentos durante o tratamento, sendo os mais frequentes os que indicam a necessidade de medicamento para condição de saúde não tratada e não adesão ao tratamento. Pacientes solteiros com diagnóstico de tuberculose há menos de 2 meses, tempo de acompanhamento de HIV/AIDS maior que 12 meses e que não são casos novos devem prioritariamente ser selecionados para acompanhamento por equipe multiprofissional de saúde para a promoção da adesão. Os problemas relacionados ao uso de medicamentos de segurança indicam maior chance de ocorrer reações adversas em indivíduos da etnia branca (Resende, et al., 2019).

Conhecer os fatores associados à ocorrência de problemas relacionados ao uso de medicamentos em pacientes coinfectados com tuberculose e HIV/AIDS propicia que a equipe de saúde implemente medidas para garantir os medicamentos indicados, efetivos e seguros para sua condição clínica e a consciência da necessidade de aderir ao tratamento proposto, ação importante para melhorar os resultados nos cuidados desses pacientes, evitando falha terapêtica e aparecimento de multirresistência (Lobo, Carvalho \& Resende, 2019).

Quando indagado sobre as ações que considerava fundamental na prática da AF voltada aos pacientes portadores de TB e HIV/AIDS, o profissional ressaltou a importância do acompanhamento contínuo do tratamento. De acordo com Silva et al. (2020), a otimização da terapia farmacológica é parte essencial no cuidado à pessoa com TB e HIV/AIDS. Os resultados deste estudo sugeriu que a avaliação das prescrições medicamentosas e a revisão da farmacoterapia por um profissional farmacêutico contribuem com a equipe multiprofissional de saúde na identificação de problemas farmacoterapêuticos, assim como na sua resolução por meio de intervenções farmacêuticas pactuadas com a equipe e com os indivíduos e direcionadas ao alcance de melhores desfechos no manejo da tuberculose, doença infecciosa que gera altos custos ao sistema de saúde.

\subsection{A assistência de farmacêutica no controle da tuberculose em pessoas em situação de rua}

As pessoas em situação de rua estão constantemente expostas a diferentes tipos de vulnerabilidade, condições de vida degradantes, o que aumenta o desafio voltado para o cuidado em saúde, necessitando de intervenções específicas para elas. Estudos demostram que essa população apresenta maiores chances de contrair tuberculose, além de apresentar taxas de comorbidades mais altas que as da população geral. No entanto, não há estudos que incorporem um conjunto mais diversos de indicadores relacionados à tuberculose e tampouco uma vasta cobertura territorial (Santos, et al., 2021).

Nesse cenário, dentre as populações mais pobres e socialmente desfavorecidas encontra-se a População em Situação de Rua, sendo as pessoas em situação de rua consideradas prioritárias para o controle da TB no Brasil. A Tabela 3, apresenta dois artigos com ano de publicação variando de 2017-2021 e apresenta as dificuldades e condutas/ações na assistência farmacêutica de casos de TB em pessoas em situação de rua, com o acompanhamento farmacoterapêutico, a fim de prevenir a não adesão ou desistência do tratamento. 
Tabela 3 - Descrição dos artigos selecionados sobre as dificuldades e condutas/ações na assistência farmacêutica aos pacientes em situação de rua com tuberculose.

\begin{tabular}{|c|c|c|c|}
\hline Referência da publicação & Tip & Dificuldades & Condutas/Ações \\
\hline $\begin{array}{l}\text { De Freitas, A. C. G. et al } \\
(2020) \text {. Tuberculose pulmonar } \\
\text { e os desafios do tratamento em } \\
\text { paciente em situação de rua: } \\
\text { relato de caso. Brazilian } \\
\text { Journal of Development, 6(10), } \\
\text { 74922-74934. }\end{array}$ & Relato de caso & $\begin{array}{l}\text { - Uso de álcool e tabaco; } \\
\text { - Situações de extrema } \\
\text { vulnerabilidade } \\
\text { econômica; } \\
\text { - Esquema terapêutico é equivocado, } \\
\text { realizado de forma irregular ou } \\
\text { interrompido precocemente, cepas } \\
\text { resistentes aos medicamentos; } \\
\text { - Resistência adquirida e/ou recidiva } \\
\text { da doença. }\end{array}$ & $\begin{array}{l}\text { - Protocolos de Tratamento, que levam } \\
\text { em consideração a existência de } \\
\text { populações de maior vulnerabilidade } \\
\text { para o adoecimento por TB e os } \\
\text { diversos tipos de resistência às } \\
\text { drogas; } \\
\text { - Ação integrada das atenções primária } \\
\text { e terciária, obtendo-se resultados } \\
\text { satisfatórios no que diz respeito à } \\
\text { adesão do tratamento e controle da } \\
\text { tuberculose na PSR }\end{array}$ \\
\hline $\begin{array}{l}\text { Valle, F. A. A. L., \& Farah, B. } \\
\text { F. (2020). A saúde de quem está } \\
\text { em situação de rua:(in) } \\
\text { visibilidades no acesso ao } \\
\text { Sistema Único de Saúde. } \\
\text { Physis: Revista de Saúde } \\
\text { Coletiva, 30(2), e300226. }\end{array}$ & $\begin{array}{l}\text { Estudo descr } \\
\text { exploratório }\end{array}$ & $\begin{array}{l}\text { - Frequência dos problemas que } \\
\text { interferem no cotidiano das ruas; } \\
\text { - O acesso à rede de assistência à } \\
\text { saúde pela } \mathrm{AB} \text {; } \\
\text { - O número de vagas/leitos foi } \\
\text { apontado como insuficiente. }\end{array}$ & $\begin{array}{l}\text { - Possibilitar o acesso a um direito } \\
\text { fundamental como o direito à saúde é } \\
\text { possibilitar, em parte, a } \\
\text { transformação da realidade das } \\
\text { pessoas que mais precisam usufruir } \\
\text { desse direito. }\end{array}$ \\
\hline
\end{tabular}

Fonte: Dados da pesquisa

A desospitalização o paciente foi acompanhado pela equipe de Consultório na Rua de Taguatinga, que realiza doses supervisionadas (TDO) e reavalia o paciente mensalmente por meio de novos exames clínicos e laboratoriais para acompanhamento da resposta ao tratamento. Desta forma, para o caso em tela, seria de extrema importância tratar este paciente como um paciente resistente à rifampicina, mesmo sem confirmação por teste de sensibilidade, uma vez que ele integra a população de risco e possui um teste rápido molecular, positivo para resistência à rifampicina. Na baciloscopia de controle referente ao quarto mês de tratamento, permaneceu abacilífero. Além disso, sua condição de rua pode dificultar a realização de um tratamento complexo e longo como o da TB novamente, em caso de recidiva. (Freitas, et al., 2020).

Os resultados do estudo de Valle \& Farah (2020) mostraram que a doença era percebida principalmente por terceiros, indicando que as pessoas em situação de rua com sinais de TB deveriam procurar um serviço de saúde. Os autores ressaltam que o tratamento da tuberculose para as pessoas em situação de rua é um desafio quando comparado ao tratamento da população em geral. Isso porque verificou-se uma baixa adesão, além das complicações provocadas pela má nutrição e comorbidades. Os medicamentos não permanecerem sob o cuidado do doente é outro fator de interferência do tratamento, já que na dinâmica das ruas é comum acontecer o roubo de pertences pessoais e até mesmo o recolhimento destes pertences pelo poder público.

\subsection{A assistência de farmacêutica no controle da tuberculose com foco na população privada de liberdade (PPL)}

O Brasil ocupa o quarto lugar com a maior população prisional do mundo, com cerca de 600 mil presos, formada principalmente por jovens negros, de baixa renda e escolaridade, e com taxas crescentes de aprisionamento, tornando-se grande fator no alto índice de transmissão da tuberculose. De acordo com Ministério da Justiça o sistema está com $161 \%$ de sua capacidade ocupada, o que significa que, em celas concebidas para custodiar dez pessoas, há em média dezesseis, superlotando as celas (Macedo, Maciel \& Struchiner, 2020).

O percentual de casos de tuberculose no sistema prisional é um grande problema para a saúde pública do Brasil, e ações decorrentes da assistência farmacêutica para o controle tendem a ser desenvolvidas para que haja uma melhora dessa doença. A Tabela 4 apresenta três (3) artigos com ano de publicação variando de 2017 à 2021, apresentando as dificuldades e condutas/ações 
na assistência farmacêutica de casos de TB, acompanhamento farmacoterapêutico para avaliar a presença da doença e melhorar a saúde de PPL.

Tabela 4 - Descrição dos artigos selecionados sobre as dificuldades e condutas/ações na assistência farmacêutica aos pacientes privado de liberdade com tuberculose.

\begin{tabular}{|c|c|c|c|}
\hline Referência da publicação & Tipo de estudo & Dificuldades & Condutas/Ações \\
\hline $\begin{array}{l}\text { Fanfa, D. C. et al. (2020). Perfil } \\
\text { epidemiológico } \\
\text { notificações dos casos de } \\
\text { tuberculose na população } \\
\text { privada de liberdade do Rio } \\
\text { Grande Do Sul, 2014-2018. } \\
\text { Revista Jovens Pesquisadores, } \\
10(1), 1-12 .\end{array}$ & $\begin{array}{l}\text { Estudo transversal } \\
\text { retrospectivo }\end{array}$ & $\begin{array}{l}\text { - A recrudescência observada no } \\
\text { estudo torna preocupante o cenário } \\
\text { da TB na PPL; } \\
\text { - coeficiente de mortalidade oscila. }\end{array}$ & $\begin{array}{l}\text { - Observou-se uma diminuição } \\
\text { progressiva nos casos do abandono } \\
\text { do tratamento; } \\
\text { - A busca ativa, intensificação da } \\
\text { vigilância epidemiológica e a } \\
\text { implementação de medidas de } \\
\text { controle foram abordagens } \\
\text { importantes na redução da incidência } \\
\text { da PPL. }\end{array}$ \\
\hline $\begin{array}{l}\text { Flora, S. M., Possagno, G. C. } \\
\text { H., Baldissera, V. D. A., \& } \\
\text { Girotto, E. (2020). Adequação } \\
\text { e validação de indicadores de } \\
\text { assistência farmacêutica no } \\
\text { sistema penitenciário. Revista } \\
\text { Baiana de Saúde Pública, } \\
44(1), 22-40 .\end{array}$ & gráfico & $\begin{array}{l}\text { - Muita discordância em relação à } \\
\text { alocação dos indicadores nas } \\
\text { dimensões estrutura, processo ou } \\
\text { resultados, e o processo de } \\
\text { validação realizado por esta } \\
\text { investigação permitiu estruturar de } \\
\text { forma adequada os indicadores } \\
\text { nessas dimensões. }\end{array}$ & $\begin{array}{l}\text { - Elaboração e validação de } \\
\text { indicadores de AF para o sistema } \\
\text { penitenciário vão ao encontro desse } \\
\text { pensamento de avaliar para a tomada } \\
\text { de decisões e melhoria da gestão de } \\
\text { recursos e processos. }\end{array}$ \\
\hline $\begin{array}{l}\text { De Oliveira, C. R., da Silva } \\
\text { Ribeiro, T., \& Dellanhese, A. P. } \\
\text { F. (2020). Medidas de } \\
\text { prevenção e controle da } \\
\text { tuberculose às pessoas privadas } \\
\text { de liberdade em presídios } \\
\text { brasileiros. Saúde Coletiva } \\
\text { (Barueri), 10(58), 3817-3830. }\end{array}$ & Revisão integrativa & $\begin{array}{l}\text { - Nos fins de semana, a entrega do } \\
\text { medicamento ficava sob a } \\
\text { responsabilidade do agente de } \\
\text { segurança; } \\
\text { - A dispensação dos medicamentos } \\
\text { era feita semanalmente por um } \\
\text { profissional de saúde, sendo o } \\
\text { tratamento auto administrado. }\end{array}$ & $\begin{array}{l}\text { - Inferiu-se que a implementação das } \\
\text { PP relacionadas às medidas de } \\
\text { prevenção e controle da TB para as } \\
\text { PPL é realizada de forma parcial. } \\
\text { - A importância da perspectiva } \\
\text { intersetorial entre o MS e MJ, } \\
\text { respeitando as competências } \\
\text { exclusivas de cada setor, a fim de } \\
\text { garantir os direitos de saúde e manter } \\
\text { os direitos humanos dos apenados. }\end{array}$ \\
\hline
\end{tabular}

Fonte: Dados da pesquisa

Das 4279 notificações no período de 2014 a 2018 destacado no estudo de Fanfa et al. (2020), predominou o sexo masculino (94,8\%), de maioria branca $(60,0 \%)$ diagnosticado com TB. Uma alta proporção de coinfecção TB/HIV foi encontrada $(18,2 \%)$. Os principais desfechos foram cura $(46,6 \%)$, transferência $(16,5 \%)$ e abandono (12,7\%). A incidência em todo período foi de 1.611,46 casos/100 mil presos, prevalência de 2.526,7 casos/100 mil presos e mortalidade de 35,43 óbitos/100 mil presos. A PPL demonstrou piores indicadores quando comparado com a população geral. A intensificação do diagnóstico prévio, bem como qualificação na assistência farmacêutica será necessária para alcançar as metas definidas pela Organização Mundial de Saúde.

O grupo foi composto neste estudo foi por dez farmacêuticos que atuam em áreas ligadas à AF. O primeiro instrumento elaborado e submetido a julgamento contemplava 114 indicadores (52 de estrutura, 49 de processo e 13 de resultado. A saúde da PPL é um problema de saúde pública emergente, sendo necessário o fortalecimento da assistência em saúde no sistema penitenciário e maior visibilidade para a AF existente, para que a recuperação da saúde seja priorizada, tornando o ambiente saudável, com segurança e racionalidade no uso de medicamentos1. Nesse sentido, ter conhecimento técnico e científico para o desenvolvimento da gestão eficiente de todo o processo é necessário, com o que o desenvolvimento e a validação dos indicadores de assistência farmacêutica penitenciária apresentados neste estudo poderão contribuir decisivamente (Flora, et al., 2020).

A pesquisa identificou carência de estudos relativos a medidas de prevenção e controle da TB no contexto carcerário brasileiro. Pode-se inferir, que a implementação das PP relacionadas às medidas de prevenção e controle da TB para as 
PPL é realizada de forma parcial. É contundente a existência de obstáculos para a implementação de forma efetiva das ações de saúde no sistema prisional, devido às más condições vida e de saúde das PPL, além das precárias condições de trabalho dos profissionais de saúde e de segurança. Ressalta-se a importância da perspectiva intersetorial entre o MS e MJ, respeitando as competências exclusivas de cada setor, a fim de garantir os direitos de saúde e manter os direitos humanos dos apenados, visando o aperfeiçoamento das estratégias voltadas à prevenção e ao controle da TB nesses estabelecimentos (Oliveira et al., 2020).

O ambiente prisional é propício para o aparecimento de diversas doenças entre elas a tuberculose. Medidas precisam ser tomadas para o controle da tuberculose no âmbito das prisões. Alguns resultados ressaltam a importância de um ambiente bem ventilado, a superlotação e a fácil propagação do bacilo em ambientes nessas condições, com isso faz com que o número de casos em presídios aumente levando grandes preocupações (Lermen, et al., 2015).

\subsection{A assistência de farmacêutica no controle da tuberculose em pacientes indígenas}

Tal aumento de demanda das populações indígenas ao consumo de medicamentos tende a expô-los aos riscos inerentes a este uso, desde a utilização indiscriminada de medicamentos (por exemplo, os antibióticos) até a ocorrência de reações adversas medicamentosas, em alguns casos, fatais. Sendo assim, o acesso da população indígena a medicamentos essenciais, eficazes, seguros e sob orientação adequada quanto ao uso, está longe de ser alcançado sem a estruturação de uma política de Assistência Farmacêutica nos setores de saúde que os assistem, visto que, a premissa do acesso e uso racional de medicamentos perpassa as diretrizes da referida política.

No contexto brasileiro, talvez com particular intensidade na região amazônica, a ideia de um posto indígena eficiente passa pela existência de uma farmácia bem estruturada. Deste modo, refletir sobre como é dada esta estruturação e que políticas e normas são adotadas para a garantia do acesso de medicamentos aos indígenas, não somente no posto indígena, mas também, nas demais localidades de atendimento assistencial pertencentes à instituição responsável pela saúde destes povos, apresenta-se inovador e simultaneamente urgente. A figura 5 apresenta três (3) artigos com ano de publicação variando de 2015 à 2021 , apresentando as dificuldades e condutas/ações na assistência farmacêutica de casos de TB e o acompanhamento farmacoterapêutico para avaliar a presença da doença e melhorar a saúde dos indígenas.

Tabela 5 - Descrição dos artigos selecionados sobre as dificuldades e condutas/ações na assistência farmacêutica aos pacientes indígenas.com tuberculose.

\begin{tabular}{|c|c|c|c|}
\hline Referência da publicação & Tipo de estudo & Dificuldades & Condutas/Ações \\
\hline $\begin{array}{l}\text { Pinheiro, D. V. (2015). } \\
\text { Panorama da tuberculose em } \\
\text { comunidades indígenas na } \\
\text { região norte do brasil. } \\
\text { FACIDER-Revista Científica, } \\
(7), 1-18\end{array}$ & $\begin{array}{l}\text { Estudo } \\
\text { bibliográfico }\end{array}$ & $\begin{array}{l}\text { - Incidência da tuberculose nessas } \\
\text { comunidades são em crianças menores } \\
\text { de } 15 \text { anos de idade; } \\
\text { - Com índices alarmantes de déficit } \\
\text { nutricional e em indivíduos do sexo } \\
\text { masculino. }\end{array}$ & $\begin{array}{l}\text { - Levantamento dos aspectos } \\
\text { epidemiológicos da tuberculose em } \\
\text { indígenas contribui e evidenciou as } \\
\text { etnias de maior acometimento e } \\
\text { incentivou, por parte das autoridades } \\
\text { governamentais. }\end{array}$ \\
\hline $\begin{array}{l}\text { De Faria, A. A. (2020). } \\
\text { Aspectos epidemiologicos da } \\
\text { tuberculose na população } \\
\text { indígena da etnia Xavante no } \\
\text { município de Barra do Garças- } \\
\text { Mato Grosso. Revista } \\
\text { Eletrônica Interdisciplinar, } \\
\text { 12(2), 082-092.. }\end{array}$ & $\begin{array}{l}\text { Estudo } \\
\text { epidemiológico, } \\
\text { retrospectivo } \\
\text { descritivo } \\
\text { documental }\end{array}$ & $\begin{array}{l}\text { - Falta de conhecimento sobre o exame } \\
\text { e despreparo dos profissionais no } \\
\text { momento da coleta; } \\
\text { - Medo, vergonha, ansiedade, } \\
\text { nervosismo e dor por parte dos } \\
\text { pacientes. }\end{array}$ & $\begin{array}{l}\text { - Ações para controlar a tuberculose } \\
\text { é necessário intensificar a vigilância } \\
\text { dos contatos e aperfeiçoar as } \\
\text { estratégias de comunicação das } \\
\text { equipes com a população indígena }\end{array}$ \\
\hline $\begin{array}{l}\text { Ferreira, T. F. et al. (2020). } \\
\text { Tendência da tuberculose em } \\
\text { indígenas no Brasil no período } \\
\text { de } 2011-2017 \text {. Ciência \& Saúde } \\
\text { Coletiva, 25, 3745-3752. }\end{array}$ & $\begin{array}{l}\text { Estudo ecológico } \\
\text { de série temporal }\end{array}$ & $\begin{array}{l}\text { - Falta de tempo, escassez de material } \\
\text { para a realização do exame, demora no } \\
\text { resultado, deficiência na infraestrutura } \\
\text { das unidades de saúde e sentimentos } \\
\text { dos indígenas de medo e vergonha em } \\
\text { realizar a coleta. }\end{array}$ & $\begin{array}{l}\text { - Importância das atividades } \\
\text { educativas junto às equipes de saúde } \\
\text { e comunidade; } \\
\text { - Enfermeiros enquadrados em um } \\
\text { novo modelo assistencial, inserido e } \\
\text { validado nos princípios básicos do } \\
\text { SUS. }\end{array}$ \\
\hline
\end{tabular}


Os índices de maior incidência da tuberculose nessas comunidades indígenas na região amazônica legal são em crianças menores de 15 anos de idade, com índices alarmantes de déficit nutricional e em indivíduos do sexo masculino. É relevante ressaltar, a suscetível condição de vida desses povos quanto ao contágio do bacilo. A TB pode ser prevenida com a utilização da vacina BCG (Bacillus Calmette-Guérin), que oferece proteção ao indivíduo contra as manifestações da TB, porém não protegem pessoas infectadas pelo bacilo que necessitam de acompanhamento rigoroso e administração de quimioterápicos de forma correta, com atuação indispensável do profissional farmacêutico. Todavia essas medidas só podem ser tomadas perante melhorias no PNCT e cooperação mútua das autoridades no intuito de reverter esse quadro da tuberculose no Brasil (Pinheiro, 2015).

Os resultados do estudo de Cunha \& Faria (2020) demonstram indicadores epidemiológicos alarmantes se comparados a de outros segmentos populacionais indígenas e não-indígenas. O coeficiente de incidência médio de tuberculose verificado nos A'uwe Xavante no decênio 2008 a 2014 foi de 1.200 por 100.000 habitantes. Foi observado registro de 66 casos de tuberculose durante o período estudado, os maiores índices de casos ocorreram em 2013 e representou 31,81\% dos casos. A prevalência ocorreu na faixa etária dos 20 aos 39 anos, com 33,33\% das notificações. O sexo masculino foi o mais acometido com 57,57\% dos casos. O estudo conclui-se que existe a necessidade de implementação de medidas de prevenção e controle voltados especificamente para a realidade dos povos indígenas.

No estudo de Ferreira et al., (2020) Foram confeccionados mapas temáticos para acompanhamento da evolução espaçotemporal da TB na população indígena em cada ano. Para o estudo da tendência utilizou-se o modelo de regressão linear generalizada de Prais-Winsten. No período em estudo, foram notificados 6.520 casos de TB em indígenas. A incidência geral de TB em indígenas no Brasil foi de 109/100.000 habitantes. Na análise por regiões do país, as maiores incidências ocorreram nas regiões Centro-Oeste, Norte e Sudeste. Na análise por UF, as maiores incidências foram encontradas no Mato Grosso, São Paulo, Rondônia, Mato Grosso do Sul, Acre, Maranhão, Pará e Rio de Janeiro. A tendência da doença nos indígenas foi estável tanto no país quanto na maioria das suas regiões e UF. A TB afeta desproporcionalmente os indígenas brasileiros e o presente estudo ao identificar regiões e UF prioritárias pode contribuir para a elaboração e fortalecimento de ações de controle mais específicas.

\section{Considerações Finais}

A assistência farmacêutica foi considerada como imprescindível para a promoção da qualidade de vida das populações chaves acometidas por tuberculose, por meio de um acompanhamento com orientações, esclarecimentos de dúvidas, escuta ativa, apoio, aconselhamento e monitorização do tratamento farmacológico para o uso racional dos medicamentos. O profissional farmacêutico foi destacado como o mais preparado para atender as necessidades medicamentosas desses pacientes chaves, pelo maior conhecimento sobre o tema, que envolve efeitos adversos, interações medicamentosas, ações do fármaco no organismo e também os benefícios do tratamento para o bem-estar do paciente.

A liberação inicial do medicamento, para o tratamento da tuberculose, fosse acompanhada da notificação do caso de tuberculose e que a farmácia participasse ativamente das notificações das doenças quando utilizassem os medicamentos. Todas as unidades tivessem a preocupação da descoberta o mais precoce possível, assim evitando a contaminação de outros pacientes e dos profissionais de saúde. A participação das farmácias comunitárias no combate à tuberculose informando os sintomas da doença, os riscos da contaminação, de diagnósticos errados e do fornecimento dos medicamentos para o tratamento gratuitamente nas unidades de saúde.

Espera-se que os resultados encontrados possam nortear ações para melhorias na qualidade dos serviços prestados pelos farmacêuticos diante da tuberculose e gerar reflexões sobre o papel desse profissional no acompanhamento do paciente com tal enfermidade. Estudos futuros que avaliem o conhecimento e a percepção da prática profissional com relação a tuberculose são fundamentais para ampliar o conhecimento nessa temática.

Para trabalhos futuros, propõe-se aprimorar a avaliação da assistência de farmacêutica no controle da tuberculose em 
populações chaves estudadas nesse trabalho acometidas, além disso, a relação ao processo de cuidado desenvolvido pelos farmacêuticos por meio da prestação dos serviços clínicos. Nesse sentido, é necessário avaliar a estrutura, o processo e, em especial, os resultados obtidos por meio dessas ações.

\section{Referências}

Alcalde, G. F. G., Santos, J. G. M., Berro, E. C., Simioni, P. U., \& Ugrinovich, L. A. (2018). Perfil epidemiológico de tuberculose em pacientes portadores de hiv. Revista Prevenção de Infecção e Saúde, 4:7519.

Araújo, E. M. N. F. D., Freitas, S. D. A., Henriques, A. H. B., Nogueira, M. F., Nogueira, J. A., \& Barrêto, A. J. R. (2020). Gestão do cuidado ao idoso com tuberculose na Atenção Primária: revisão integrativa. Revista Brasileira de Geriatria e Gerontologia, 23(06): ):e190269.

Barreira, D. (2018). Os desafios para a eliminação da tuberculose no Brasil. Epidemiologia e Serviços de Saúde, $27:$ e00100009.

Belardinelli, L. B., Franzmann, U. T., Alves, C. N., \& Fontenele, R. M. (2020). Perfil epidemiológico dos casos de tuberculose em pessoas privadas de liberdade no Brasil. Research, Society and Development, 9(11), e72291110243-e72291110243.

Bertolozzi, M. R., Takahashi, R. F., França, F. O. D. S., \& Hino, P. (2019). A ocorrência da tuberculose e sua relação com as desigualdades sociais: estudo de revisão Integrativa na Base PubMed. Escola Anna Nery, 24(1): e20180367.

Cortez, A. O., Melo, A. C. D., Neves, L. D. O., Resende, K. A., \& Camargos, P. (2021). Tuberculose no Brasil: um país, múltiplas realidades. Jornal Brasileiro de Pneumologia, 47 (2): e20200119.

Da Silva, B. N., de Abreu Temoteo, R. C., Véras, G. C. B., \& Silva, C. R. D. V. (2019). Fatores predisponentes de tuberculose em indivíduos privados de liberdade: revisão integrativa. Arquivos de Ciências da Saúde, 26(1), 67-71.

Da Silva, D. B., da Costa, G. S., Rosa, L. F. B., dos Santos Guilherme, M., de Oliveira, S. A., \& de Souza Cavalcanti, R. L. (2017). Assistência farmacêutica a pacientes com tuberculose pulmonar: uma revisão integrativa. Revista Presença, 3(7), 83-106.

Da Silva, I. P., Marques, L. I., Penha, R. M., \& Polisel, C. G. (2020). Problemas relacionados à farmacoterapia e intervenções farmacêuticas em indivíduos com tuberculose. Revista Brasileira de Pesquisa em Saúde, 22(1), 60-70.

Da Silva, M. C., \& de Lima, J. G. (2021). Contribuição farmacêutica na logística de medicamentos e acompanhamento clínico de pacientes com tuberculose e hanseníase na atenção primária à saúde. Revista Eletrônica Acervo Saúde, 13(2), e6398-e6398.

De Andrade, L. K., Belotti, N. C. U., Cury, M. R. D. C. O., de Souza, A. R., Pedro, H. D. S. P., \& Chimara, E. (2018). Mycobacterium tuberculosis e micobactérias não tuberculose em paciente com síndrome da imunodeficiência adquirida e câncer: relato de caso. Arquivos de Ciências da Saúde da UNIPAR, 22(1): 49-53.

De BRITO, A. C., PEZAREZI, S. C., \& dos SASAKI, N. S. G. M. (2017). Análise das publicações sobre tuberculose no sistema prisional. Revista Corpus Hippocraticum, 1(1): 1-10.

De Faria, A. A. (2020). Aspectos epidemiologicos da tuberculose na população indígena da etnia Xavante no município de Barra do Garças-Mato Grosso. Revista Eletrônica Interdisciplinar, 12(2), 082-092.

Felipe, T. V., de Macedo Pinto, A. C., Ribeiro, P. B. P., \& do Nascimento Martins, N. V. (2021). Tuberculose no sistema prisional: avaliação do conhecimento de servidores do Sistema Penitenciário em um município da Amazônia Legal. Research, Society and Development, 10 (2), e45110212669-e45110212669.

Ferri, A. O., Aguiar, B., Wilhelm, C. M., Schmidt, D., Fussieger, F., \& Picoli, S. U. (2014). Diagnóstico da tuberculose: uma revisão. Revista Liberato, 15(24), $145-154$.

Giacometti, M. T., de Andrade, L. G., Pugliese, F. S., \& da Silva, M. S. (2021). Atenção Farmacêutica No Tratamento De Tuberculose. Revista Ibero-Americana de Humanidades, Ciências e Educação, 7(8), 296-309.

Giergowicz, F. B., \& Mahmud, I. C. (2020). A população privada de liberdade e a tuberculose: perfil epidemiológico em Porto Alegre/RS. Sci. med.(Porto Alegre, Online), 30(1): 37951-37951.

Gonçalves, A. F., Cristo, J., \& Junior, A. P. (2019). Perfil epidemiológico de casos de tuberculose no município de Paragominas-PA. Revista Saúde e Meio Ambiente, 9(2): 34-48.

Jacobs, M. G., \& Pinto Junior, V. L. (2020). Caracterização da tuberculose drogarresistente no Brasil, 2014. Epidemiologia e Serviços de Saúde, $28:$ e2018294.

Lermen, H. S., Gil, B. L., Cúnico, S. D., \& Jesus, L. O. D. (2015). Saúde no cárcere: análise das políticas sociais de saúde voltadas à população prisional brasileira. Physis: Revista de Saúde Coletiva, 25 (3): 905-924.

Macedo, L. R., Maciel, E. L. N., \& Struchiner, C. J. (2020). Fatores associados à tuberculose na população privada de liberdade no Espírito Santo. Revista de Saúde Pública, 54(67):1-12

Miranda, R. D. N. A., Gonçalves, R. S. L., Morais, R. M., de Souza, R. G., \& Vieira, C. R. S. F. (2019). Caracterização nutricional de pacientes com HIV/AIDS coinfectados ou não com tuberculose internados no hospital universitário em Belém, estado do Pará. Revista Eletrônica Acervo Saúde, (28): e976-e976.

Muniz, H. V. L., da Silva Neto, A. F., Sales, L. A. T., Soares, T. R., \& Alves, M. S. (2020). Desenvolvimento de primers in silico de Mycobacterium tuberculosis da região 16s para o diagnóstico da tuberculose. Interfaces Científicas-Saúde e Ambiente, 8(2): 77-86. 
Nicoletti, G. P., de Araújo Antunes, A., Gurgel, J. A. R., da Silva Costa, S., \& Brandão, G. H. A. (2020). A importância do profissional farmacêutico no processo de cura da tuberculose. Brazilian Journal of Development, 6(11), 85213-85238.

Oliveira, G. D. C. A., Silva, A. C. S. S., Regazzi, I. C. R., Nasser, M. D. R. M., Brust, R. S., \& Knupp, V. M. D. A. O. (2021). Epidemiological profile of the population with tuberculosis in the Rio de Janeiro State, RPCFO, 13: 197-204.

Paiva, B. L., Nogueira, L. M. V., Rorigues, I. L. A., Basta, P. C., Ferreira, Â. M. R., \& Caldas, S. P. (2019). Modelo preditivo de determinantes socioeconômicos da tuberculose em população indígena do estado do pará, brasil. Cogitare Enfermagem, 24: e64835.

Ralaizara, B., Andriamandrimbisoa, A., Chevallier, E., \& Mattern, C. (2020). Tuberculosis and HIV's management: A health seeking behaviour Analysis of Incarcerated Patients. European Journal of Public Health, 30(5): 166-848.

Ramos, J., Wakoff-Pereira, M. F., Cordeiro-Santos, M., Albuquerque, M. D. F. M. D., Hill, P. C., Menzies, D., \& Trajman, A. (2018). Conhecimento e percepção de médicos e enfermeiros em três capitais brasileiras com alta incidência de tuberculose a respeito da transmissão e prevenção da doença. Jornal Brasileiro de Pneumologia, 44: 168-170.

Rodrigues, F. D. F., Aquino, R., \& Medina, M. G. (2018). Avaliação dos serviços farmacêuticos na Atenção Primária à Saúde no cuidado ao paciente com tuberculose. Saúde em Debate, 42, 173-187.

Rodrigues, F. D. F., Aquino, R., \& Medina, M. G. (2018). Avaliação dos serviços farmacêuticos na Atenção Primária à Saúde no cuidado ao paciente com tuberculose. Saúde em Debate, 42, 173-187.

Santos, A. C. E. D., Brunfentrinker, C., Pena, L. D. S., Saraiva, S. D. S., \& Boing, A. F. (2021). Análise e comparação dos desfechos do tratamento de tuberculose na população em situação de rua e na população geral do Brasil. Jornal Brasileiro de Pneumologia, 47(2):e20200178.

Silva, T. O., Vianna, P. J. D. S., Almeida, M. V. G., Santos, S. D. D., \& Nery, J. S. (2021). População em situação de rua no Brasil: estudo descritivo sobre o perfil sociodemográfico e da morbidade por tuberculose, 2014-2019. Epidemiologia e Serviços de Saúde, 30 (1): e2020566.

Soares, S. V., Picolli, I. R. A., \& Casagrande, J. L. (2018). Pesquisa bibliográfica, pesquisa bibliométrica, artigo de revisão e ensaio teórico em administração e contabilidade. Administração: ensino e pesquisa, 19(2), 308-339.

Tavares, E. C. F., da Silva, L. C., dos Santos Tenório, S., da Silva Sena, D., de Alencar, G. B., Ribeiro, T. K. S., ... \& Monteiro, E. L. (2021). Fatores determinantes e a incidência de tuberculose na população indígena: Uma revisão integrativa da literatura. Research, Society and Development, 10(6), e8110615417e8110615417.

Winter, B. C. D. A., \& Grazinoli Garrido, R. (2017). La tuberculosis en el cárcel: un retrato de las adversidades del sistema prisional brasileño. Medicina Legal de Costa Rica, 34(2), 20-31.

World Health Organization (2020b). Global Tuberculosis Report 2020, Genebra. Relatório: https://www.who.int/teams/global-tuberculosis- programme/tbreports.

World health statistics (2020a). Monitoring health for the SDGs, sustainable development goals.. https://apps.who.int/iris/handle/10665/332070.

Zorzi, S. S., Campos, R. B. C., Quadros, S. R. D., Zilly, A., Palha, P. F., Gabardo, B. M. A., \& Silva-Sobrinho, R. A. (2019). Tuberculose em população indígena autodeclarada no Estado do Paraná. Rev. bras. ciênc. saúde, 23(1): 57-64. 